\title{
Mielomalácia hemorrágica progressiva em 14 cães $^{1}$
}

\author{
Débora de M. Zilio² e Mônica V. Bahr Arias²*
}

\begin{abstract}
Zilio D.M \& Bahr Arias M.V. 2013. [Progressive hemorrhagic myelomalacia in 14 dogs.] Mielomalácia hemorrágica progressiva em 14 cães. Pesquisa Veterinária Brasileira 33(2):219-228. Departamento de Clínicas Veterinárias, Universidade Estadual de Londrina, Rodovia Celso Garcia Cid, PR 445 Km 380, Campus Universitário, Londrina, PR 86051-990, Brazil. E-mail: vicky@uel.br

Progressive hemorrhagic myelomalacia (PHM) is a rare and fatal disorder which is characterized by acute and progressive ischemic necrosis of the parenchyma of the spinal cord, leading to its liquefaction. It may occur after intervertebral disc extrusion, spinal trauma or fibrocartilaginous embolism. The aim of this study was to evaluate cases of progressive hemorrhagic myelomalacia in dogs in the Veterinary Hospital of Universidade Estadual de Londrina between 2000 and 2011, through the analysis of medical records and following of cases. There were certain criteria to include a patient in this study, such a history of paraplegia with upper motor neuron signs, worse of signs progressing to flaccid tetraplegia, progressive clinical changes and/or changes in complementary exams. There were analyzed several aspects, such as epidemiological (breed, age and sex), clinical (progress of clinical and neurological signs), laboratory (cerebrospinal fluid analysis - CSF), radiographic (conventional radiography and contrasted) and elapsed time since the onset of clinical signs until death or euthanasia. The most affected breed was Teckel (43\%), the average age was 5.04 years and the neurological syndrome observed initially was the thoracolumbar syndrome grade V. Another commons signs observed were hyperpathia and cranial progression of decreased cutaneous trunci reflex. In seven dogs the cause of the PHM was the thoracolumbar intervertebral disc disease, in a dog the cause was spinal cord trauma, in two dogs PHM was due to lymphoma and in four dogs the likely cause was intervertebral disc disease. CSF analysis, myelography changes and progress of clinical and neurological examinations were extremely important to diagnose PHM. Six animals progressed to tetraplegia and four dogs had already flaccid tetraplegia at the initial care. In four other patients, the identification of signs suggestive of PHM before this progression has led to indication for euthanasia. The prognosis is poor and causes animal suffering, so the clinician should be aware of the history of paraplegia with subsequent change of upper motor neuron syndrome to lower motor neuron, cranial decreased reflex panniculus and presence of abdominal breathing. Some alterations in complementary exams found in this study may also help in early diagnosis, as xanthochromic CSF with increased protein, erythrocytes and pleocytosis. Spinal cord edema and the presence of contrast within the nervous tissue together with clinical signs and CSF alterations are suggestive of PHM.
\end{abstract}

INDEX TERMS: Spinal cord, nervous system, myelomalacia, dogs.

RESUMO.- A mielomalácia hemorrágica progressiva (MHP) é uma afecção rara e fatal, em que ocorre necrose aguda isquêmica e progressiva do parênquima da medula espinhal,

\footnotetext{
${ }^{1}$ Recebido em 28 de setembro de 2011.

Aceito para publicação em 6 de junho de 2012.

2 Departamento de Clínicas Veterinárias, Universidade Estadual de Londrina (UEL), Campus Universitário, Rodovia Celso Garcia Cid, PR 445 Km 380, Londrina, PR 86051-990, Brasil. *Autor para correspondência: vicky@uel.br
}

levando à liquefação da mesma. Pode ocorrer após extrusão do disco intervertebral, trauma medular ou embolismo fibrocartilaginoso. Este estudo teve como objetivo avaliar casos de mielomalácia hemorrágica progressiva em cães atendidos no Hospital Veterinário da Universidade Estadual de Londrina entre os anos 2000 e 2011, realizando-se a análise dos prontuários de atendimento e acompanhamento dos casos. Os animais do presente estudo atendiam a alguns critérios de inclusão, como histórico de paraplegia com sinais de 
neurônio motor superior, piora dos sinais progredindo para tetraplegia flácida, alterações clínicas progressivas e/ou alterações nos exames complementares. Foram analisados os aspectos epidemiológicos (raça, idade e sexo), clínicos (evolução dos sinais clínicos e neurológicos), laboratoriais (análise do líquido cefalorraquidiano), radiográficos (radiografias simples e contrastadas) e o tempo decorrido desde o início dos sinais clínicos até óbito ou eutanásia. A raça Teckel foi a mais acometida (43\%), a média de idade foi de 5,04 anos e no atendimento inicial a síndrome toracolombar grau $\mathrm{V}$ foi a alteração mais encontrada, além de hiperpatia e progressão cranial da diminuição do reflexo cutâneo do tronco. Em sete cães a causa da MHP foi a doença do disco intervertebral toracolombar, em um cão a causa foi o trauma medular, em dois cães a MHP foi decorrente de linfoma e em quatro cães a causa provável foi doença de disco intervertebral. Alterações na análise do líquido cerebroespinhal, na mielografia e na evolução dos sinais clínicos e neurológicos foram extremamente importantes para diagnosticar a MHP. Seis animais progrediram para tetraplegia e quatro cães já apresentavam tetraplegia flácida no atendimento inicial. Em outros quatro pacientes, a identificação de sinais sugestivos de MHP antes desta progressão levou à indicação de eutanásia. Como o prognóstico é ruim e ocasiona sofrimento ao animal, o clínico deve estar atento ao histórico de paraplegia com posterior mudança da síndrome de neurônio motor superior para neurônio motor inferior, diminuição do reflexo cutâneo do tronco cranialmente e presença de respiração abdominal, sendo que algumas alterações em exames complementares encontradas neste trabalho também podem auxiliar no diagnóstico precoce da MHP, como o líquido cerebroespinhal xantocrômico com aumento de proteínas, hemácias e pleocitose. Na mielografia o edema medular e a presença de contraste no interior do tecido nervoso, frente às alterações clínicas e liquóricas, são sugestivas de MHP.

TERMOS DE INDEXAÇÃO: Medula espinhal, sistema nervoso, mielomalácia, cães.

\section{INTRODUÇÃO}

A mielomalácia hemorrágica progressiva (MHP) é uma desordem neurovascular (Braund 2003a), rara e fatal, que pode ocorrer após a extrusão do disco intervertebral, trauma medular e embolismo fibrocartilaginoso (Braund 2003b). A lesão uma combinação de infarto isquêmico e hemorrágico do parênquima da medula espinhal (De Lahunta 1983), caracterizada pela liquefação da mesma (Dewey 2006), hemorragia nos espaços epidural, subaracnóideo e parênquima da medula espinhal (Lu et al. 2002, Inzana 2003).

A MHP tem início no local da lesão ou trauma inicial, progredindo em direção ascendente e descendente (Braund 2003b). A etiologia da MHP não é conhecida, mas estudos sugerem que catecolaminas liberadas pelo tecido nervoso atuem nos vasos intramedulares ou ocorra vasoespasmo das leptomeninges ao haver liberação de substâncias como glutamato, histamina, serotonina, dopamina e norepinefrina (De Lahunta \& Glass 2009), o que pode ser deflagrado pela hemorragia no momento da lesão inicial (Summers et al. 1995).
Os sinais clínicos iniciam-se com paraplegia aguda e reflexos espinhais aumentados nos membros pélvicos. Em exame posterior, após 48 a 72 horas, a paraplegia torna-se flácida, com atonia dos músculos dos membros pélvicos, flacidez da cauda e dilatação do ânus, com ausência do reflexo perineal (De Lahunta 1983, De Lahunta \& Glass 2009). À medida que a lesão progride cranial e caudalmente, há tetraplegia, musculatura abdominal e membros torácicos flácidos e presença de dor à manipulação em região torácica ou cervical. A respiração torna-se diafragmática e pode ocorrer síndrome de Horner bilateral. A síndrome medular identificada inicialmente se altera, sendo que uma lesão inicial diagnosticada como de neurônio motor superior (NMS) para os membros posteriores torna-se do tipo neurônio motor inferior (NMI) (De Lahunta 1983, Sharp \& Wheeler 2005). Com a progressão ascendente da MHP ocorre perda progressiva do reflexo cutâneo do tronco e os membros torácicos também passam a apresentar alterações compatíveis com lesão do tipo NMI (Denny \& Butterworth 2006). 0 óbito do animal ocorre sete a 10 dias a partir do início dos sinais e é causado por paralisia respiratória (De Lahunta 1983, Braund 2003b). Outros sinais como apatia, anorexia, vômito, hipotensão, toxemia e hiperestesia também podem ser observados (Sharp \& Wheeler 2005). Estes últimos autores sugerem a eutanásia assim que os sinais clínicos são reconhecidos, para evitar maior sofrimento e morte por paralisia respiratória, pois infelizmente não há tratamento para essa afecção.

O objetivo deste estudo foi avaliar entre os anos de 2000 e 2011, cães atendidos com sinais de mielomalácia hemorrágica progressiva no Hospital Veterinário (HV) da Universidade Estadual de Londrina (UEL), considerando os aspectos epidemiológicos (raça, idade e sexo), clínicos (evolução dos sinais clínicos e neurológicos), laboratoriais (análise do líquido cefalorraquidiano) e radiográficos (radiografias simples e contrastadas).

\section{MATERIAL E MÉTODOS}

Foram analisados os prontuários e realizou-se o acompanhamento de cães com sinais de mielomalácia hemorrágica progressiva, atendidos entre os anos de 2000 e 2011 no Hospital Veterinário (HV) da Universidade Estadual de Londrina (UEL). Os critérios de inclusão foram o histórico inicial de paraplegia aguda, ocorrendo piora dos sinais com progressão para tetraplegia flácida, curso clínico progressivo e/ou alterações nos exames complementares ou necropsia.

Os dados utilizados para este estudo foram: raça, sexo e idade; alterações clínicas, neurológicas e grau de disfunção neurológica no atendimento inicial; evolução dos sinais clínicos e neurológicos; hemograma, análise do líquido cefalorraquidiano; achados radiográficos em exames simples e contrastados (mielografia); etiologia e o tempo de evolução desde os sinais iniciais até óbito ou realização de eutanásia. 0 diagnóstico de mielomalácia foi realizado com base nos sinais clínicos e neurológicos, por meio de necropsia ou pela observação da mesma durante a cirurgia de hemilaminectomia. Durante a cirurgia, se houvesse a visualização de alteração difusa da coloração da medula espinhal para rosa/ arroxeado por mais de um segmento medular, era realizada a durotomia com auxílio de uma agulha hipodérmica para verificar a presença de mielomalácia difusa, caracterizada pela perda da 
consistência normal da medula espinhal (Amsellem et al. 2003, Sharp \& Wheeler 2005, Blaser et al. 2012).

No exame clínico, tanto no atendimento inicial quanto na evolução dos sinais, os parâmetros observados foram principalmente temperatura, frequência cardíaca e respiratória e o tipo de respiração. Quanto ao exame neurológico, os animais foram avaliados em relação à consciência, locomoção, reações posturais, nervos cranianos, reflexos espinhais, reflexo cutâneo do tronco, reflexo perineal, controle da micção, presença ou ausência de dor profunda e presença ou ausência de dor à palpação da coluna vertebral. A classificação do grau de disfunção da síndrome toracolombar (T3L3), considerando a severidade da lesão no atendimento inicial, foi realizada de acordo com Griffiths (1982), em que I significa dor toracolombar, sem alterações neurológicas; II - paraparesia ambulatória; III - paraparesia não ambulatória; IV - paraplegia com retenção ou incontinência urinária e V-paraplegia com ausência de dor profunda associada à retenção ou incontinência urinária. O diagnóstico da presença ou ausência da sensibilidade dolorosa profunda nos pacientes paraplégicos foi realizado aplicando-se a pressão de uma pinça hemostática no periósteo dos dígitos e observando se o animal manifestava uma resposta consciente, como vocalização ou movimentação da cabeça em direção ao estímulo doloroso, indicando que foi transmitido pela medula espinhal ao encéfalo (Amsellem et al. 2003, Sharp \& Wheeler 2005). Foi considerada síndrome de Horner a presença de no mínimo miose pupilar e protrusão da terceira pálpebra, acrescido ou não de enoftalmia e ptose palpebral (Braund 1994).

Radiografias simples sob tranquilização com meperidina na dose de $3 \mathrm{mg} / \mathrm{kg}$ e acepromazina na dose de $03 \mathrm{mg} / \mathrm{kg}$, e coleta de líquido cerebroespinhal e mielografia sob anestesia geral também foram realizadas, quando indicado, para exclusão de outras causas e localização da lesão para realização de hemilaminectomia, nos casos em que inicialmente não havia suspeita da ocorrência de mielomalácia. Hemograma completo foi realizado em 10 animais.

0 acesso para a coleta de líquido cerebroespinhal e injeção de contraste foi a cisterna magna, após realização de anestesia geral com propofol na dose de $5 \mathrm{mg} / \mathrm{kg}$, entubação e manutenção com anestesia inalatória com isoflurano (Sharp \& Wheller 2005). Logo após a coleta de dois $\mathrm{mL}$ de líquido cerebroespinhal, que era imediatamente enviado ao laboratório para análise, injetava-se lentamente $0,5 \mathrm{~mL} / \mathrm{kg}$ do meio de contraste iohexol 300mg/ ml. Os pacientes eram mantidos por cinco a dez minutos com o tronco elevado a $45^{\circ}$, e a seguir era realizado o exame radiográfico látero-lateral e ventro-dorsal. Nos casos em que, após a injeção do contraste na cisterna magna e realização das radiografias, não foi possível identificar o local correto da compressão, devido ao edema intenso da medula espinhal,, caracterizado pela falha de preenchimento do espaço subaracnoide sobre vários espaços intervertebrais, realizou-se injeção de $0,3 \mathrm{~mL} / \mathrm{kg}$ de contraste na região lombar entre as vértebras L5-L6 ou L6-L7, segundo técnica descrita por Sharp \& Wheller (2005). Considerou-se líquido cerebroespinhal alterado a contagem de mais de 5 leucócitos $/ \mathrm{mm}^{3}$ ou proteína total maior que $25 \mathrm{mg} / \mathrm{dL}$ para o líquido cerebroespinhal coletado da cisterna magna (Sharp \& Wheller 2005). Quando havia pleocitose realizava-se a contagem diferencial dos leucócitos para definir se a pleocitose era mista, linfocítica ou neutrofílica. Não foi administrado corticóide nem antes nem depois da realização destes exames.

Os animais foram distribuídos em dois grupos: casos confirmados por necropsia/observação de mielomalácia no trans-operatório de hemilaminectomia (Grupo $\mathrm{A} ; \mathrm{n}=7$ ) e casos com diagnóstico presuntivo, sem confirmação por necropsia/observação de mielomalácia no trans-operatório de hemilaminectomia (Grupo B; n=7). Neste trabalho, foram estudados 14 cães que atendiam aos critérios de inclusão.

\section{RESULTADOS E DISCUSSÃO}

Os dados referentes à raça, sexo, idade, peso, tempo para atendimento desde o início do quadro e histórico, estão detalhados no Quadro 1. Foram atendidos nove cães de raça e cinco cães sem raça definida (SRD), sendo a raça Teckel a mais acometida (6/14 [43\%]). A idade variou entre dois a oito anos (média de 5,1 anos) e o peso entre 3,5 e $27 \mathrm{~kg}$ (média de 10,87 kg). As causas de MHP foram extrusão do disco em 11 animais (sete casos confirmados e quatro presumidos), linfoma em dois e trauma exógeno causado por atropelamento em um cão. A MHP, que pode ocorrer como sequela após lesão aguda da medula espinhal (Lu et al. 2002), afeta qualquer raça e idade, porém, devido à relação existente entre a doença do disco intervertebral (DDIV) tipo I e a mielomalácia, raças predispostas à extrusão do disco como o Teckel, Poodle e Shih Tzu, são mais comumente afetadas (Inzana 2003), como observado no presente estudo. A idade média dos animais também é condizente com a idade de ocorrência de DDIV (Sharp \& Wheeler 2005, Denny \& Butterworth 2006) e a prevalência deste tipo de complicação em cães com paraplegia devido à DDIV e perda da dor profunda é de 5\% (Summers et al. 1995) a 11\% (Sharp \& Wheeler 2005, Platt et al. 2006, Taylor 2009).

Os animais foram atendidos entre um e quatro dias após o início dos sinais clínicos, sendo a paraplegia súbita sem trauma exógeno o sinal clínico mais comum relatado no histórico do paciente, com exceção de um cão que havia sido atropelado, ocorrendo consequente trauma medular. Esta afecção pode ocorrer entre poucas horas a um dia após o insulto inicial, seja ele DDIV, trauma exógeno ou in-

Quadro 1. Raça, sexo, idade, peso, tempo para atendimento

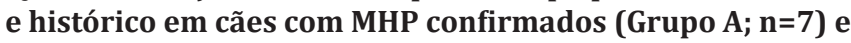
em cães com diagnóstico presuntivo de MHP (Grupo B; n=7) atendidos no HV/UEL entre 2000 e 2011

\begin{tabular}{|c|c|c|}
\hline & Grupo A & Grupo \\
\hline \multicolumn{3}{|l|}{ Raça } \\
\hline Teckel: & 1 & 5 \\
\hline Sem raça definida: & 3 & 2 \\
\hline Australian Cattle Dog: & 1 & 0 \\
\hline Poodle: & 1 & 0 \\
\hline Shih tzu: & 1 & 0 \\
\hline \multicolumn{3}{|l|}{ Sexo } \\
\hline Fêmea: & 4 & 3 \\
\hline Macho: & 3 & 4 \\
\hline \multicolumn{3}{|l|}{ Idade } \\
\hline 1 a 5 anos: & 3 & 4 \\
\hline 6 a 10 anos: & 4 & 3 \\
\hline MÉDIA = 5,07 & & \\
\hline \multicolumn{3}{|l|}{ Peso } \\
\hline < 5 kg: & 2 & 0 \\
\hline 5 a $20 \mathrm{~kg}:$ & 4 & 7 \\
\hline$>20 \mathrm{~kg}:$ & 1 & 0 \\
\hline \multicolumn{3}{|l|}{ Tempo entre ocorrência e atendimento } \\
\hline 0 a 8 horas: & 1 & 0 \\
\hline 8 a 24 horas: & 1 & 0 \\
\hline >24 horas: & 5 & 7 \\
\hline \multicolumn{3}{|l|}{ Histórico } \\
\hline Paraplegia aguda: & 4 & 4 \\
\hline $\begin{array}{l}\text { Ataxia de membros pélvicos evoluindo para } \\
\text { paraplegia: }\end{array}$ & 2 & 2 \\
\hline Atropelamento: & 0 & 1 \\
\hline Dor cervical: & 1 & 0 \\
\hline
\end{tabular}


farto fibrocartilaginoso (Braund 2003a), mas nem sempre a paraplegia é o sinal clínico inicial, e sim dor de origem indeterminada, letargia e desconforto (Platt et al. 2006).

No exame clínico inicial, oito animais não apresentaram alterações, mas dois animais manifestaram hipertermia, dois hipotermia, um bradicardia e um taquicardia (Quadro 2). Segundo Inzana (2003), Hoerlein (1978) e Lecouteur \& Grandy (2010), a hipertermia pode ser decorrente da hemorragia subaracnóide secundária à necrose da microvasculatura da medula espinhal, levando a dor intensa. A hiperestesia espinhal difusa foi constatada em sete pacientes, realizando-se palpação dos processos espinhosos em direção caudocranial e avaliando-se a resposta do paciente (Sharp \& Wheeler 2005). Outros sinais que podem ser observados são apatia, anorexia, vômito e hipotensão (Sharp \& Wheeler 2005). As consequências fisiopatológicas da dor podem justificar a taquicardia (Muir III 2009a). Ao acompanhar-se a evolução do quadro, observou-se o aparecimento de hipertermia em um animal e de hipotermia em três, sinal este não descrito na literatura consultada, e que pode estar relacionado com a toxemia (Sharp \& Wheeler 2005).

Já no exame neurológico inicial, os principais achados foram síndrome toracolombar grau $\mathrm{V}$ em sete animais e grau IV em três. Embora a mielomalácia possa afetar cães com DDIV grau V, segundo Sharp \& Wheeler (2005), ocasionalmente cães com síndrome toracolombar grau IV também podem desenvolver mielomalácia, como constatado no presente trabalho, passando a apresentar paraplegia grau V. Quatro animais apresentaram tetraplegia com ausência de dor profunda nos membros pélvicos no atendimento inicial, e flacidez dos membros torácicos, com histórico de paraplegia iniciado um a sete dias (média de 3,75 dias) antes do atendimento. Os sinais clínicos dependem da localização da lesão; contudo, a maioria dos animais

Quadro 2. Principais alterações clínicas e sinais neurológicos em cães confirmados com MHP (Grupo A; n=7) e em cães com diagnóstico presuntivo de MHP (Grupo B; $\mathbf{n = 7}$ ) atendidos no HV/UEL entre 2000 e 2011

\begin{tabular}{lcc}
\hline & Grupo A & Grupo B \\
\hline Alterações clínicas no atendimento inicial & & 3 \\
Sem alterações & 5 & 1 \\
Hipertermia & 1 & 2 \\
Hipotermia & 0 & 1 \\
Bradicardia & 0 & 1 \\
Taquicardia & 0 & 1 \\
Pulso fraco & 0 & 1 \\
Taquipnéia & 0 & 1 \\
Respiração abdominal & 0 & 1 \\
Mucosas pálidas visíveis & 1 & 1 \\
Mucosas congestas & 0 & 0 \\
Linfoadenomegalia generalizada & 1 & 4 \\
Principais sinais neurológicos observados no atendimento inicial \\
Síndrome toracolombar grau V: & 3 & 1 \\
Síndrome toracolombar grau IV: & 2 & 2 \\
Tetraplegia com ausência de dor profunda nos & 2 & \\
membros pélvicos: & & 3 \\
Reflexo cutâneo do tronco diminuído: & 2 & 3 \\
Hiperestesia espinhal: & 4 & 0 \\
Síndrome de Schiff-Scherrington: & 2 & 1 \\
Síndrome de Horner bilateral: & 1 & 2 \\
Esfíncter anal relaxado: & 1 & \\
Obnubilação: & & \\
& & 1
\end{tabular}

Quadro 3. Evolução dos sinais clínicos e neurológicos e tempo de evolução desde sinais iniciais até óbito ou eutanásia em cães confirmados com MHP (Grupo A; n=7) e em cães com diagnóstico presuntivo de MHP (Grupo B; $n=7$ ) atendidos no HV/UEL entre 2000 e 2011

\begin{tabular}{llc}
\hline & Grupo A & Grupo B \\
\hline $\begin{array}{l}\text { Evolução dos sinais clínicos e neurológicos } \\
\text { Hipertermia }\end{array}$ & 0 & 1 \\
Hipotermia: & 1 & 2 \\
Respiração abdominal & 2 & 4 \\
Tetraplegia & 2 & 4 \\
Reflexos espinhais ausentes & 3 & 4 \\
Síndrome de Horner bilateral & 0 & 2 \\
Esfíncter anal relaxado & 2 & 3 \\
Perda progressiva do reflexo cutâneo do tronco & 3 & 3 \\
cranialmente & & \\
Perda da sensibilidade profunda & 2 & 2 \\
Hiperpatia & 3 & 1 \\
Alteração vocalização & 0 & 1 \\
Estupor & 0 & \\
Tempo de evolução desde sinais iniciais até a mor- & & \\
te ou eutanásia & & 2 \\
1 a 5 dias & 6 & 5 \\
6 a 11 dias & 1 & \\
MÉDIA = 5,1 & &
\end{tabular}

afetados inicialmente apresenta sinais clínicos indicativos de mielopatia entre T3 e L3, com sinais de NMS nos membros pélvicos (Lecouteur \& Grandy 2010), evoluindo para tetraplegia como observado nos animais deste trabalho, devido à progressão da necrose medular (Braund 2003a, DeLahunta \& Glass 2009).

Síndrome de Schiff-Sherrington foi observada em dois cães (Quadro 2). Esta síndrome pode surgir se houver envolvimento da medula espinhal entre os segmentos medulares T3 e L3 (Lecouteur \& Grandy 2010), devido ao comprometimento dos neurônios que inibem os neurônios extensores dos membros torácicos (DeLahunta \& Glass 2009), porém esta síndrome não foi uma observação frequente no presente estudo, provavelmente pela rápida progressão da lesão, que leva a hipotonia e arreflexia dos membros torácicos (DeLahunta \& Glass 2009). A progressão da perda do reflexo cutâneo do tronco cranialmente é um sinal característico da evolução da MHP (Sharp \& Wheeler 2005), e foi observada em seis animais (Quadro 3), e, em um animal paraplégico este reflexo estava ausente caudal à vértebra T5 já no atendimento inicial. Segundo Muguet-Chanoit et al. (2011), cães com DDIV e progressão cranial da perda do reflexo cutâneo do tronco tem 145 vezes mais chance de desenvolver MHP do que cães com regressão caudal do reflexo, que estaria associado a probabilidade de melhora clínica. Como este reflexo é segmentar, permite a avaliação da função sensorial de segmentos medulares individualmente (Muguet-Chanoit et al. 2011).

Um animal apresentou obnubilação, e segundo Lu et al. (2002), o estado mental diminuído pode ser observado em casos de MHP. Respiração abdominal foi constatada em um animal no atendimento inicial e em seis animais durante o acompanhamento dos casos (Quadros 1 e 3). Na evolução do quadro, devido ao envolvimento dos segmentos medulares cervicais, podem ocorrer hipoventilação e apneia devido à paralisia respiratória, com evidente respiração abdominal 
(Sharp \& Wheeler 2005), caso os nervos intercostais e frênicos sejam afetados em sua origem nos segmentos medulares cervicotorácicos (Inzana 2003).

A síndrome de Horner bilateral também pode ocorrer (Lecouteur \& Grandy 2010), e foi observada em dois animais no atendimento inicial e em mais dois na evolução clínica, indicando comprometimento dos corpos celulares pré-ganglionares simpáticos localizados na substância cinzenta dos segmentos medulares cervicotorácicos (De Lahunta \& Glass 2009). Assim, a progressão ascendente ou descendente das deficiências neurológicas, tetraparesia, síndrome de Horner, perda progressiva do reflexo cutâneo do tronco cranialmente (Denny \& Butterworth 2006) e uma linha de analgesia que também avança cranialmente (Inzana 2003), observados nos animais estudados, foram fundamentais para diagnosticar a MHP. Já os sinais indicativos de progressão da mielomalácia em direção aos segmentos lombosacrais são o desenvolvimento de paraplegia flácida com atonia dos membros pélvicos e cauda, esfíncter anal relaxado, analgesia da cauda, períneo, ânus e membros pélvicos (Braund 2003a, De Lahunta \& Glass 2009). O esfíncter anal relaxado com analgesia e cauda flácida (Fig.1B) foi observado em três animais no atendimento inicial e em mais cinco durante a evolução da MHP.

Existem controvérsias quanto ao tempo de evolução da MHP entre os autores consultados. De acordo com Sharp \& Wheeler (2005) e Taylor (2009), esta condição normalmente se desenvolve após cinco dias da ocorrência da paralisia inicial, com intervalo entre um e 10 dias (Sharp \& Wheeler 2005). Segundo De Lahunta \& Glass (2009), entre

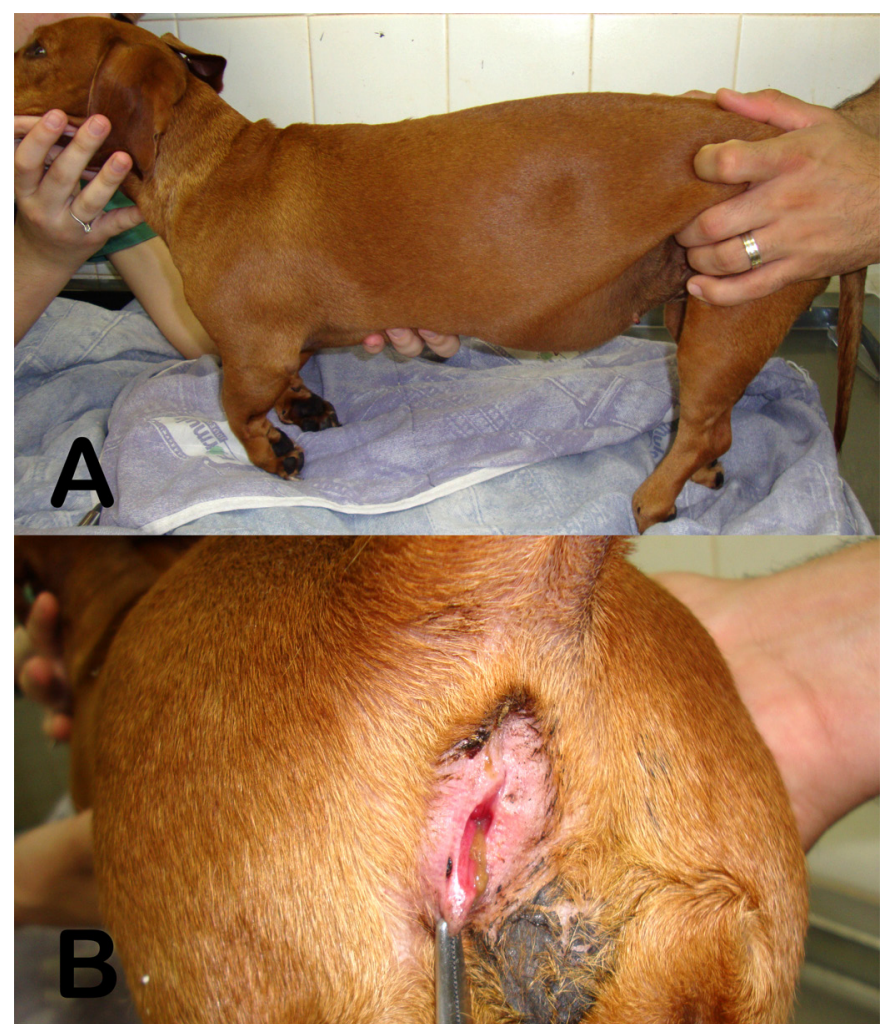

Fig.1. Cadela com sinais sugestivos de MHP. (A) Tetraplegia flácida. (B) Analgesia cutânea e esfíncter anal relaxado, não responsivo ao estímulo com pinça hemostática. um e três dias após a extrusão, a necrose isquêmica progride cranial e caudalmente. Os mesmos autores também relataram a ocorrência de MHP em um cão 12 dias após o início de paraplegia aguda. Neste estudo, foi avaliado o tempo de ocorrência de MHP desde o atendimento inicial no Hospital Veterinário até a morte ou eutanásia. Observou-se que a MHP se desenvolveu em uma média de 5,1 dias após o início dos sinais (Quadro 3), com um intervalo entre um e 11 dias, sendo que em um animal a eutanásia foi realizada no atendimento inicial, pois o quadro estava bem avançado.

As principais alterações observadas no hemograma de cães com DDIV foram linfopenia (25\%) e trombocitose (25\%). Em quatro animais não foram observadas alterações. A linfopenia pode ser um sinal de estresse e também ocorre em animais com dor (Muir III 2009b). Em cães com MHP o hemograma geralmente é normal no início do quadro, podendo ocorrer um desvio à esquerda degenerativo depois que a condição se estabelece, causado pela necrose massiva da medula espinhal (Inzana 2003), o que não foi constatado nos animais do presente estudo. Já o aumento do número de plaquetas pode ser decorrente de um processo inflamatório ou estresse (Reagan 1997).

O líquido cefalorraquidiano foi coletado em oito animais, sendo que em um deles o líquido cerebroespinhal não foi analisado, porém encontrava-se turvo. Em um cão não foram encontradas alterações no líquido cerebroespinhal. Em seis pacientes (Quadro 4 e 5) observou-se no líquido cefalorraquidiano a presença de 965 a 19.200 hemácias/ $\mathrm{mm}^{3}$ (média de 6440,3 hemácias), e pleocitose, com um intervalo de 24 a 374 leucócitos/ $\mathrm{mm}^{3}$ (média de 121,4 leucócitos). Dentre os animais com pleocitose, observou-se misto de linfócitos e segmentado em dois, pleocitose linfocítica

Quadro 4. Principais alterações encontradas na análise do líquido cerebroespinhal, radiografia simples e mielografia em cães confirmados com MHP (Grupo A; n=7) e em cães com diagnóstico presuntivo de MHP (Grupo B; $\mathbf{n = 7}$ ) atendidos no HV/UEL entre 2000 e 2011

\begin{tabular}{lcc}
\hline & Grupo A & Grupo B \\
\hline Alterações na análise do líquido cerebroespinhal & $(\mathrm{n}=5)$ & $(\mathrm{n}=2)$ \\
Aspecto turvo & 5 & 1 \\
Presença de hemácias & 5 & 1 \\
Coloração avermelhada & 3 & 0 \\
Coloração xantocrômica & 2 & 1 \\
Aumento de proteína & 4 & 1 \\
Pleocitose ${ }^{\mathrm{a}}$ mista & 2 & 0 \\
Pleocitose linfocitária & 1 & 0 \\
Pleocitose neutrofílica & 1 & 1 \\
Sem alterações: & 0 & 1 \\
Alterações na radiografia simples & $(\mathrm{n}=5)$ & $(\mathrm{n}=5)$ \\
Calcificação de discos intervertebrais & 2 & 4 \\
Diminuição do espaço intervertebral & 1 & 0 \\
Fratura compressiva em vértebra lombar, com des- & 0 & 1 \\
locamento dorsal e compressão do canal medular & & \\
Alterações na mielografia & $(\mathrm{n}=2)$ & $(\mathrm{n}=3)$ \\
Interrupção da coluna de contraste: & 2 & 3 \\
Contraste no interior do parênquima: & 1 & 2 \\
Falha de preenchimento: & 1 & 1
\end{tabular}

${ }^{a}$ Pleocitose $=$ mais de 5 células $/ \mathrm{mm}^{3}$, Pleocitose mista $=$ porcentagem igual de neutrófilos e células mononucleares, Pleocitose linfocitária = maior porcentagem de linfócitos, Pleocitose neutrofílica $=$ predomínio de neutrófilos 
Quadro 5. Resultado do exame de líquido cerebroespinhal em cães com MHP confirmada (Animais 1 a 5) e presumida (Animal 6)

\begin{tabular}{|c|c|c|c|c|c|c|}
\hline Animal & Cor & Aspecto & Leucócitos/mm3 & Diferencial & Hemácias/mm3 & Proteína mg/dL \\
\hline 1 & Avermelhado & Turvo & 106 & $\begin{array}{c}50 \% \text { segm. } \\
50 \% \text { linf. }\end{array}$ & 19.200 & - \\
\hline 2 & Xantocrômico & Turvo & 75 & $\begin{array}{l}94 \% \text { linf. } \\
6 \% \text { segm. }\end{array}$ & 2286 & 49,1 \\
\hline 3 & Avermelhado & Turvo & 28 & $\begin{array}{l}52 \% \text { segm. } \\
48 \% \text { linf. }\end{array}$ & 7509 & 92,3 \\
\hline 4 & Avermelhado & Turvo & Normal & - & 3050 & 86 \\
\hline 5 & Xantocrômico & Turvo & 24 & 91\% segm. & 965 & 52 \\
\hline 6 & Xantocrômico & Turvo & 374 & 91\% segm & 5632 & 74,4 \\
\hline
\end{tabular}

em um e neutrofílica em dois. 0 aumento de proteína foi observado em quatro animais, com variações entre 49,1 e 92,3mg/dL (média de 70,76mg/dL). Na análise do líquido cerebroespinhal de cães com MHP pode haver pleocitose leve a moderada, com misto de células, resultante de necrose ou inflamação secundária à mielomalácia (Terlizzi \& Platt 2009) e também altos níveis de proteína (Sharp \& Wheeler 2005). A pleocitose foi constatada em 87,5\% (6/7) dos animais em que este exame foi realizado, e ao contrário da literatura consultada, observou-se também pleocitose linfocítica e neutrofílica. Além da pleocitose, também foi observado aumento no número de hemácias. Segundo Okada et al. (2010), que avaliaram 12 cães com MHP em um período de 11 anos, o líquido cerebroespinhal xantocrômico é um sinal precoce de MHP.

Nas radiografias simples, a calcificação de discos intervetebrais "in situ" foi a alteração mais constatada. Estas radiografias não devem ser usadas como o único meio diagnóstico da DDIV, somente para descartar outras causas de paraplegia, como fraturas, luxações e osteólise (Sharp \& Wheeler 2005). No animal com histórico de atropelamento, observou-se fratura compressiva na primeira vértebra lombar com deslocamento dorsal. Já na mielografia, realizada em cinco cães, os principais achados foram interrupção da coluna de contraste, seis a oito vértebras acima do local da extrusão do disco em quatro animais, indicando edema medular, e contraste no interior do parênquima em três pacientes (Quadro 3), sendo estas alterações observadas concomitantemente em dois animais. Certas alterações na mielografia podem ser indicativas de MHP, como o edema medular e a presença de meio de contraste no interior do parênquima da medula espinhal, entretanto não existem trabalhos relatando estas alterações (Lu et al. 2002). 0 edema medular pode ocorrer não só devido à mielomalácia, mas a neoplasias, inflamação, infarto fibrocartilaginoso e hemorragia (Sharp \& Wheeler 2005), entretanto quando o edema estende-se por mais de cinco corpos vertebrais, o prognóstico parece ser reservado (Lu et al. 2002). Apesar de existirem controvérsias sobre esta afirmação (Lu et al. 2002), em cães com MHP a falha de preenchimento devido ao edema foi bastante extensa (Fig.2). 0 contraste no interior do tecido nervoso pode ser decorrente de falha técnica, por injeção inadvertida do mesmo (Lu et al. 2002), ou devido à secção da medula após fratura com grande deslocamento vertebral (Sharp \& Wheeler 2005). No presente estudo, o contraste no interior do tecido foi visibilizado próximo ao local da extrusão na transição toracolombar, longe do local da injeção do contraste pela cisterna magna ou pela região lombar, indicando realmente alteração no parênquima medular.

Quatro animais foram submetidos à hemilaminectomia, pois inicialmente não haviam sinais clínicos sugestivos de mielomalácia. Observou-se mielomalácia difusa em um paciente, focal em outro, equimoses na medula em um cão e um animal não apresentava alterações. Nestes dois últimos pacientes os sinais clínicos desenvolveram-se após a cirurgia. 0 paciente com mielomalácia focal apresentava alterações sugestivas no líquido cerebroespinhal e presença de contraste no interior do parênquima medular. Segundo Amsellem et al. (2003), o diagnóstico de mielomalácia no trans-operatório é realizado após a durotomia, na qual observa-se a medula com alteração da coloração e consistência pastosa focal ou difusa. Em estudo realizado por Scott \& McKee (1999), 9\% dos cães submetidos à laminectomia desenvolveram mielomalácia progressiva no período pós-operatório, enquanto outros $9 \%$ foram submetidos à eutanásia no trans-operatório devido à mielomalácia difusa observada. A MHP nem sempre é visível durante a cirurgia, e alguns animais podem apresentar mielomalácia focal, existindo a possibilidade de recuperação clínica (Sharp \& Wheeler 2005). Assim, seria precipitado realizar a eutanásia no trans-operatório, recomendando-se avaliar minuciosamente o paciente em busca de sinais de MHP, realizando-se exame neurológico sequencial caso não haja alterações no líquido cerebroespinhal e na mielografia. Embora haja controvérsias sobre os benefícios da durotomia, indica-se sua realização em caso de suspeita de MHP, pois permite a avaliação da integridade do tecido nervoso confirmando ou excluindo a MHP (Blaser et al. 2012).

Em dois pacientes, a MHP estava relacionada a linfoma: uma cadela de sete anos, sem raça definida e $27 \mathrm{~kg}$ foi trazida para atendimento apresentando tetraplegia flácida, após quadro de dor cervical, dificuldade para movimentar a cabeça e paraplegia súbita. Apresentou piora do quadro, com dores intensas em coluna cervical, cervicotorácica e toracolombar. Ao exame clínico, observou-se respiração abdominal, aumento do linfonodo pré-escapular esquerdo, síndrome de Horner bilateral e esfíncter anal relaxado. Nos exames laboratoriais não foram constatadas alterações. Devido à suspeita de MHP, foi submetida à eutanásia. $\mathrm{Na}$ necropsia foram observados nódulos difusos, firmes e ulcerados na mucosa estomacal e na abertura do canal vertebral observou-se mielomalácia em toda a extensão. Pela citologia e histologia dos linfonodos foi confirmado linfo- 
ma. Outro cão, com três anos de idade, da raça Australian Cattle Dog e $17 \mathrm{~kg}$ foi trazido com histórico de paraplegia súbita há um dia. No exame inicial, apresentou mucosas visivelmente pálidas e linfonodomegalia generalizada. No exame neurológico, constatou-se piora do quadro inicial, que progrediu rapidamente para tetraplegia flácida. Foi observado também sensibilidade profunda ausente em membros pélvicos, esfíncter anal relaxado e síndrome de Horner bilateral. Nos exames laboratoriais, observou-se leucocitose com desvio (45.700 leucócitos) e hematócrito diminuído (19,1\%). Assim, a eutanásia foi realizada devido à suspeita de MHP. Na citologia dos linfonodos observou-se alta celularidade com figuras de mitose frequentes, o que foi sugestivo de linfoma. Na abertura do canal vertebral, foi observado mielomalácia em toda a medula espinhal. Nestes dois casos confirmados como linfoma, não observaram-se na necropsia alterações vertebrais compatíveis com extrusão de disco intervertebral.

Um caso de mielomalácia associada a linfoma epidural em um equino foi relatado por Rousseaux et al. (1989), provavelmente devido à compressão e isquemia, ocasionada pela obstrução do fluxo venoso pelo próprio tumor ou pelos tecidos circundantes comprimidos. 0 cavalo apresen- tou leucopenia algumas semanas antes da piora do quadro, ao contrário de um dos cães diagnosticado com linfoma no presente estudo, em que foi observado leucocitose. Em cães relata-se encefalomalácia e mielomalácia decorrentes de linfoma intravascular, mas no trabalho consultado não é descrito se a mielomalácia era difusa (McDonough et al 2002). Em humanos relata-se a ocorrência de infarto medular como apresentação inicial da linfomatose maligna intravascular por obstrução das artérias espinhais (Liu et al. 2009) e da leucemia linfocítica, sendo que neste último caso foi relatado mielomalácia difusa com necrose dos segmentos medulares cervicais, torácicos e lombares (Avilés et al. 1989). Segundo De Lahunta \& Glass (2009), a MHP também pode ser vista em cães com neoplasia extradural e hemorragia epidural difusa sem DDIV, entretanto não foram localizados relatos de MHP relacionados a linfoma.

A necropsia foi realizada em cinco animais, com visualização da destruição e liquefação da medula espinhal, sendo que dois animais eram os pacientes com linfoma. Nos outros três animais a causa da MHP foi a DDIV, cujo disco extruso foi visualizado no canal vertebral. A MHP caracteriza-se por uma coloração arroxeada da medula espinhal estendendo-se por vários segmentos medulares, e após a
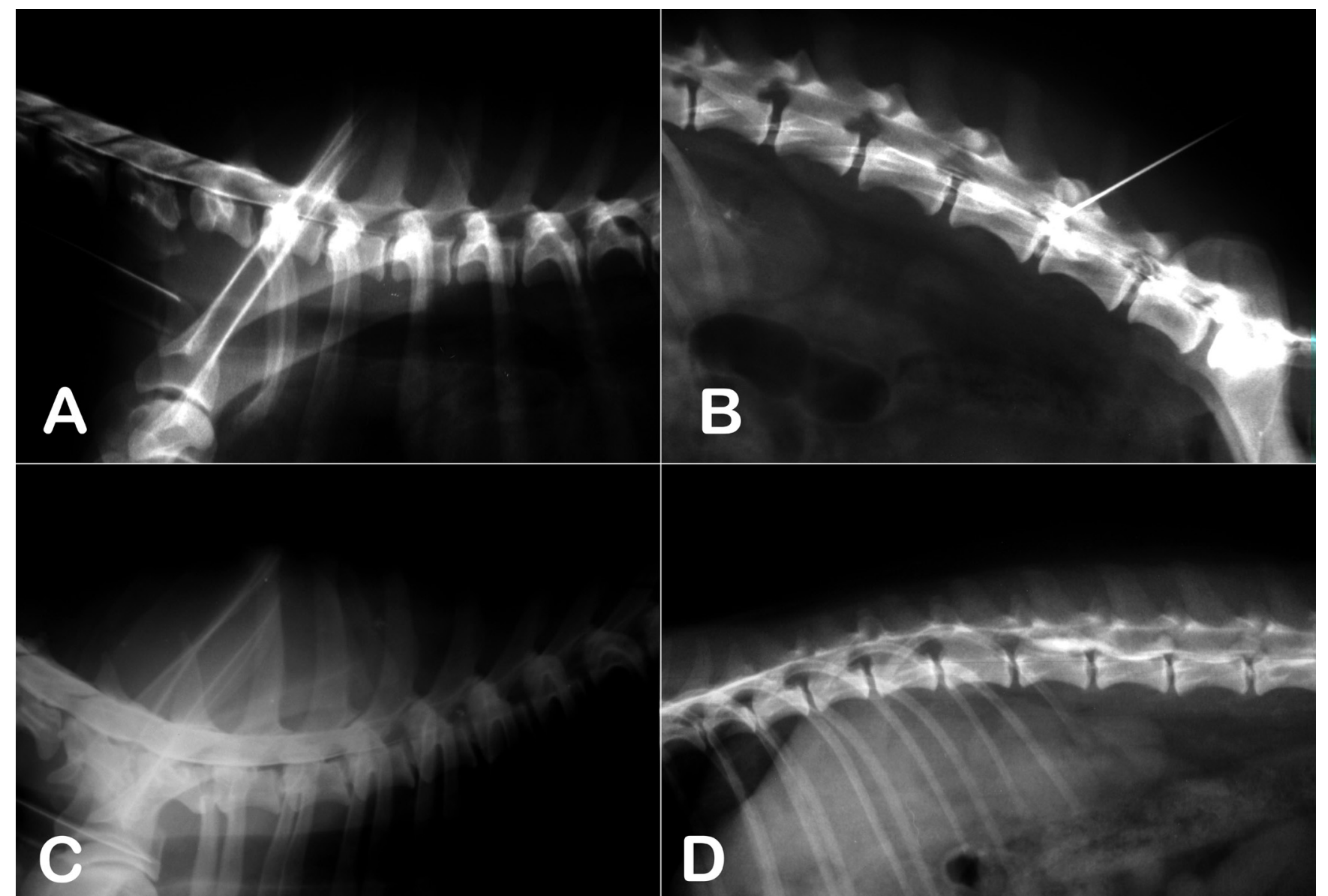

Fig.2. Mielografia. (A) Interrupção da coluna de contraste sobre a vértebra T2, após injeção do contraste pela cisterna magna. (B) Mesmo cão de (A)após a injeção de contraste via lombar. Não há progressão do contraste em direção cranial, indicando que o edema da medula espinhal se estende desde vértebras torácicas até vértebras lombares. (C) Interrupção da coluna de contraste a partir de T3. A extrusão do disco era entre as vértebras T11-T12, indicando edema medular intenso (D) Presença de contraste no interior do parênquima medular em segmento medular sobre a vértebra L2 em outro animal. 

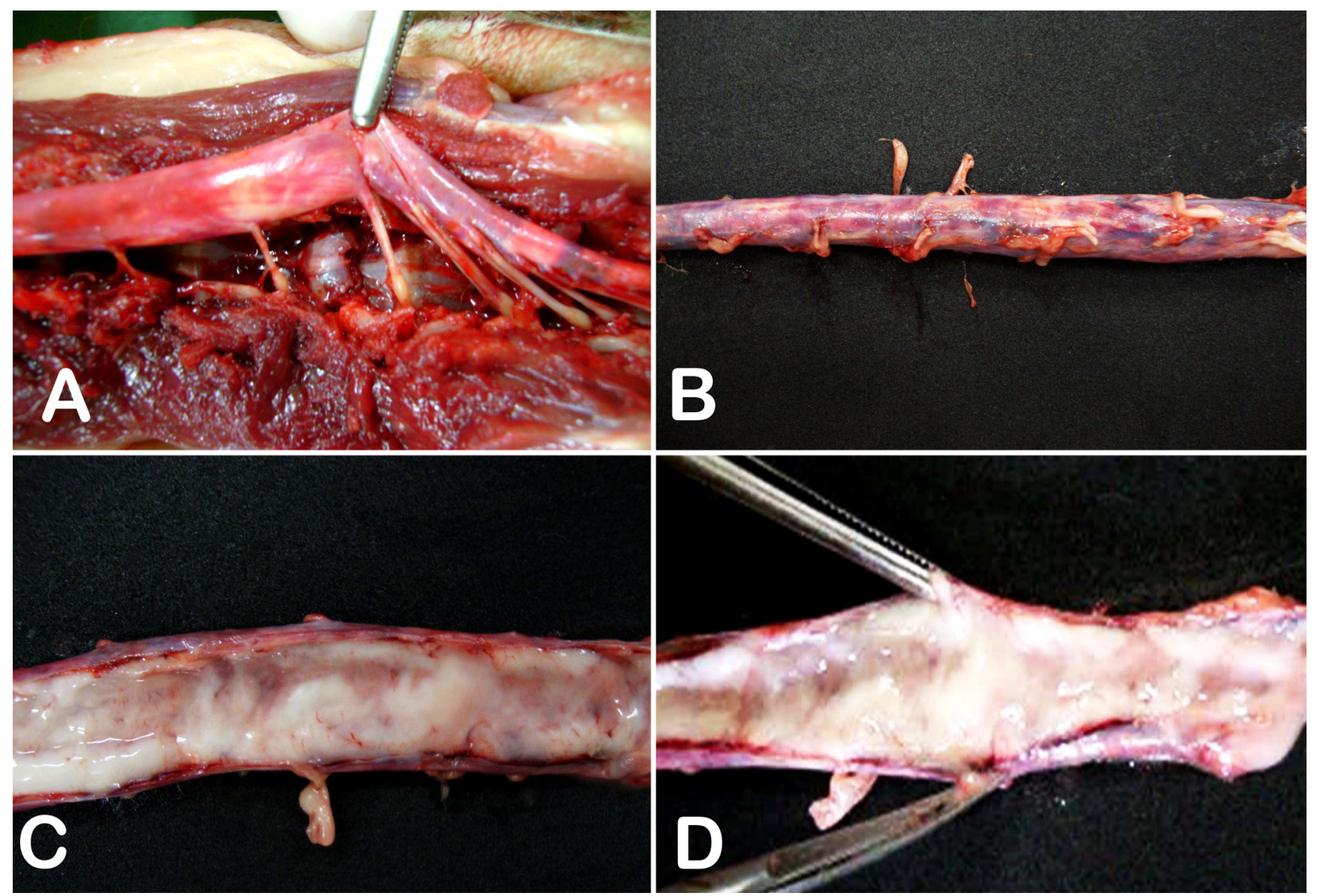

Fig.3. Medula espinhal após abertura do canal vertebral durante a necropsia. (A) Coloração rosa-arroxeada estendendo-se por vários segmentos medulares. (B) Mesmo aspecto na face ventral da medula após retirada do canal vertebral. (C) e (D) Aspecto pastoso da medula espinhal após a abertura da dura-máter, com perda da estrutura e consistência normal.

incisão da dura-máter, observa-se a saída de um material de consistência pastosa por toda a extensão da medula afetada (Fig.3) (Sharp \& Wheeler 2005). 0 comprometimento da arquitetura do tecido nervoso é tão grande que a divisão anatômica entre substância branca e cinzenta é perdida (Braund 2003a).

Quando a MHP desenvolve-se não existem chances de recuperação (Taylor 2009), apenas em alguns casos raros em que a mielomalácia pode não progredir a ponto de envolver a medula cervical; contudo, a lesão medular e as deficiências neurológicas serão permanentes (Lecouteur \& Grandy 2010). Como não existem tratamento clínico ou cirúrgico efetivos, a eutanásia é recomendada (Lecouteur \& Grandy 2010), pois quando a necrose atinge os segmentos medulares cervicais os animais morrem de asfixia com extremo desconforto (De Lahunta \& Glass 2009). No presente estudo a eutanásia foi recomendada em todos os casos, assim que suspeitou-se de mielomalácia. Porém, dois proprietários não aceitaram a indicação e levaram o animal, ocorrendo o óbito em casa em um paciente, e o outro foi trazido de volta ao HV para realização da eutanásia devido à piora do quadro.

Uma possível explicação para a ocorrência de MHP leva em consideração o suprimento sanguíneo para os segmentos espinhais toracolombares (De Lahunta \& Glass 2009).
Estudos em cães mostram que, normalmente, existe uma artéria espinhal calibrosa similar à de humanos na altura das vértebras L5 ou L6, que supre vários segmentos espinhais, cranial e caudalmente. Possivelmente, em cães que desenvolvem mielomalácia progressiva essa artéria se encontra cranialmente, no local onde a extrusão do disco é relatada em casos de mielomalácia, ou seja, T10 a L3. 0 dano a essa artéria tem o potencial de comprometer um grande segmento da medula espinhal, e os efeitos secundários dessa lesão podem levar à necrose dos segmentos menos vascularizados, em sentido cranial e caudal (De Lahunta \& Glass 2009).

Segundo Hoerlein (1978), os danos que levam à ocorrência de MHP são causados por vasoespasmo resultante da hemorragia subaracnóide, com consequente falha da circulação colateral, liberação de substâncias vasoconstritoras, trombose de alguns vasos e liberação de aminas e catecolaminas. 0 processo autocatalítico gera necrose ascendente e descendente dos neurônios motores e das fibras sensitivas, e assim, a medula isquêmica necrosa rapidamente e provoca destruição completa do tecido nervoso (McLaughlin 2004). A hemorragia vascular progride longitudinalmente porque os vasos no sistema nervoso central possuem pouco ou nenhum tecido muscular para promover vasoconstrição e coagulação, causando 
uma progressiva destruição do tecido medular (Hoerlein 1978). De acordo com Platt et al. (2006), a morte celular na substância cinzenta pode ocorrer após quatro horas de ocorrência da lesão inicial, e a área de necrose pode se expandir em poucos dias.

0 clínico deve estar atento ao histórico de paraplegia com posterior mudança da lesão do tipo NMS para NMI, diminuição do reflexo cutâneo do tronco cranialmente, envolvimento de membros anteriores, (Toombs \& Waters 2003), conforme observado em doze animais do presente estudo e mudança de padrão respiratório para respiração abdominal, sendo, atualmente, a eutanásia a única alternativa, visto que o mecanismo desta afecção não está elucidado. Infelizmente, não há como prever quais cães serão acometidos pela mielomalácia (Toombs \& Waters 2003). Neste estudo, foi possível observar que a MHP pode ser identificada não só por meio do exame neurológico sequencial, levando em consideração a progressão dos sinais clínicos e neurológicos característicos desta afecção, mas também pela análise do líquido cerebroespinhal e mielografia. Nem sempre a MHP ocorrerá somente após extrusão do disco intervertebral ou trauma medular, podendo ser decorrente também de linfoma. Assim, deve-se buscar sinais clínicos e laboratoriais indicativos de linfoma em cães com sinais de MHP.

\section{CONCLUSÕES}

O acompanhamento de 14 cães com MHP confirmada ou presumida permitiu concluir que:

As causas de MHP foram a doença do disco intervertebral toracolombar, o trauma medular e o linfoma.

A raça Teckel foi a mais acometida, a média de idade foi de 5,04 anos e no atendimento inicial a síndrome toracolombar grau $\mathrm{V}$ foi a alteração mais encontrada, entretanto cães com síndrome toracolombar grau IV também evoluiram para MHP.

A progressão da perda do reflexo cutâneo do tronco cranialmente foi um sinal característico da evolução da MHP.

Além destes sinais, a progressão para tetraplegia flácida, a presença de síndrome de Horner, desenvolvimento de paraplegia flácida com atonia dos membros pélvicos e cauda, esfíncter anal relaxado com analgesia e cauda flácida foram também sinais clínicos característicos de MHP.

Foram bastante significativas as alterações na análise do líquido cerebroespinhal, como a presença de xantocromia, pleocitose e aumento de proteína.

Na mielografia o edema medular e a presença de meio de contraste no interior do tecido nervoso também foram sugestivas de MHP.

Agradecimentos.- Aos residentes do HV/UEL, pela colaboração no atendimento, discussão dos casos e preenchimento das fichas.

\section{REFERÊNCIAS}

Amsellem P.M., Toombs J.P., Laverty P.H. \& Breur G.J. 2003. Loss of deep pain perception following thoracolumbar intervertebral disk herniation in dogs: treatment and prognosis. Comp. Cont. Educ. Pract. Vet. 25:266274.
Blaser A., Lang J., Henke D., Doherr M.G., Adami C. \& Forterre F. 2012. Influence of durotomy on laser-doppler measurement of spinal cord blood flow in chondrodystrophic dogs with thoracolumbar disk extrusion. Vet. Surg. 41:221-227.

Braund K.G. 1994. Clinical Syndromes in Veterinary Neurology. $2^{\text {nd }}$ ed. Mosby, St Louis. 477p.

Braund K.G. 2003a. Neurovascular disorders. In: Vite C.H. (Ed.), Braund's Clinical Neurology in Small Animals: Localization, diagnosis and treatment. International Veterinary Information Service (www.ivis.org). Ithaca, NY. 23p. Disponível em <http://www.ivis.org/advances/Vite/ braund25/chapter_frm.asp?LA=1> Acessado em maio de 2011.

Braund K.G. 2003b. Neurological syndromes. In: Vite C.H. (Ed.), Braund's Clinical Neurology in Small Animals: Localization, diagnosis and treatment. International Veterinary Information Service (www.ivis.org). Ithaca, NY. 14p. Disponível em <http://www.ivis.org/advances/Vite/ braund1/chapter_frm.asp?LA=1> Acessado em maio de 2011.

De Lahunta A. 1983. Lower motor neuron: spinal nerve, general somatic efferent system, p.82-83. In: De Lahunta A. (Ed.), Veterinary Neuroanatomy and Clinical Neurology. $2^{\text {nd }}$ ed. W.B. Saunders, St Louis.

De Lahunta A. \& Glass E. 2009. Lower motor neuron: Spinal nerve, general somatic efferent system, p.119-120. In: De Lahunta A. \& Glass E. (Eds), Veterinary Neuroanatomy and Clinical Neurology. $3^{\text {rd }}$ ed. W.B. Saunders, St Louis.

Denny H.R. \& Butterworth S.J. 2006. Doença do disco toracolombar, p.193194. In: Denny H.R. \& Butterworth (Eds), Cirurgia Ortopédica em Cães e Gatos. 4aㅡ ed. Roca, São Paulo.

Dewey C.W. 2006. Mielopatias: doenças da medula espinhal, p.163-195. In: Ibid. (Ed.), Neurologia de Cães e Gatos: guia prático. Roca, São Paulo. $352 \mathrm{p}$.

Griffiths I.R. 1982. Spinal disease in the dog. In Practice 4:44-52.

Hoerlein B.F. 1978. Canine Neurology: diagnosis and treatment. $3^{\text {rd }}$ ed. W.B. Saunders, Philadelphia. 593p.

Inzana K.D. 2003. Mielomalácia hemorrágica difusa (hematomielia), p.975. In: Tilley, L.P. \& Smith F.W.K.Jr. (Eds), Consulta Veterinária em 5 minutos: espécies canina e felina. $2^{\mathrm{a}}$ ed. Manole, São Paulo.

Lecouteur R. \& Grandy J.L. 2010. Diseases of the spinal Cord. In: Ettinger S.J. \& Feldman E.C. (Eds), Textbook of Veterinary Internal Medicine. $7^{\text {th }}$ ed. W.B. Saunders, St Louis. 2208p.

Liu H., Koyanagi I., Chiba H., Wanibuchi M. \& Honmou O. 2009. Spinal cord infarct as the initial clinical presentation of intravascular malignant lymphomatosis. J. Clin. Neurosci. 16:570-573.

Lu D., Lamb C.R. \& Targett M.P. 2002. Results of myelography in seven dogs with mielomalacia. Vet. Radiol. Ultrasound 43:326-330.

McDonough S.P., Van Winkle T.J., Valentine B.A., VanGessel Y.A. \& Summers B.A. 2002. Clinicopathological and immunophenotypical features of canine intravascular lymphoma (Malignant angioendotheliomatosis). J. Comp. Pathol. 126:277-288.

McLaughlin R. 2004. Doença do disco intervertebral, p.386. In: Harari J. (Ed.), Segredos em Cirurgia de Pequenos Animais. Artmed, Porto Alegre.

Muguet-Chanoit A.C., Olby N.J., Lim J.H., Gallagher R., Niman Z., Dillard S., Campbell J., Early P., Mariani C.L., Muñana K.R., Freeman C., Platt S.R., Kent M., Giovanella C. \& Longshore R.C. 2012. The Cutaneous Trunci Muscle Reflex: A predictor of recovery in dogs with acute thoracolumbar myelopathies caused by intervertebral disc extrusions. Vet. Surg. 41:201-206.

Muir III W.M. 2009a. Fisiologia e fisiopatologia da dor, p.13-41. In: Gaynor J.S. \& Muir III W.W. (Eds), Manual de Controle da Dor em Medicina Veterinária. $2^{\underline{a}}$ ed. Editora MedVet, São Paulo.

Muir III W.M. 2009b. Dor e estresse, p.42-56. In: Gaynor J.S. \& Muir III W.W. (Eds), Manual de Controle da Dor em Medicina Veterinária. 2ª ed. Editora MedVet, São Paulo.

Okada M., Kitagawa M., Ito D., Itou T., Kanayama K. \& Sakai T. 2010. Magnetic resonance imaging features and clinical signs associated with presumptive and confirmed progressive myelomalacia in dogs: 12 cases (1997-2008). J. Am. Vet. Med. Assoc. 10:1160-1165. 
Platt S.R., McConnell J.F. \& Bestbier M. 2006. Magnetic resonance imaging characteristics of ascending hemorrhagic myelomalacia in a dog. Vet. Radiol. Ultrasound 47:78-82.

Reagan W.J. 1997. Thrombocyitosis, p.280 In: Tilley L.P. \& Smith Jr F.W.K. (Eds), The 5 Minute Veterinary Consult: Canine and feline. $1^{\text {st }}$ ed. Willians and Wilkins, Baltimore.

Rousseaux C.G., Doige C.E. \& Tuddenham T.J. 1989. Epidural lymphosarcoma with myelomalacia in a seven-year-old Arabian gelding. Can. Vet. J. 30(9):751-753.

Scott H.W. \& McKee W.M. 1999. Laminectomy for 34 dogs with thoracolumbar intervertebral disc disease and loss of deep pain perception. J. Small Anim. Pract. 40:417-422.

Sharp N.J.H. \& Wheeler S.J. 2005. Thoracolumbar disc disease, p.128-159.
In: Sharp N.J.H. \& Wheeler S.J. (Eds), Small Animal Spinal Disorders: Diagnosis and surgery. $2^{\text {nd }} e d$. Mosby, Philadelphia.

Summers B.A., Cummings J.F. \& De Lahunta A. 1995. Injuries to the central nervous system, p.203-204. In: Summers B.A., Cummings J.F. \& De Lahunta A. (Eds), Veterinary Neuropathology. Mosby, St Louis.

Taylor S.M. 2009. Disorders of the spinal cord, p.1076-1077. In: Nelson R.W. \& Couto C.G. (Eds), Small Animal Internal Medicine. $4^{\text {th }}$ ed. Mosby, Philadelphia.

Terlizzi R.D. \& Platt S.R. 2009. The function, composition and analysis of cerebrospinal fluid in companion animals. Part II. Analysis. Vet. Journal 180:15-32.

Toombs J.P \& Waters D.J. 2003. Intervertebral disc disease, p.1193-1209. In: Slatter D. (Ed.), Textbook of Small Animal Surgery. $3^{\text {rd }}$ ed. W.B. Saunders, Philadelphia. 